\title{
COMUNIDADES DE MACROINVERTEBRADOS EDÁFICOS EN DIFERENTES SISTEMAS DE USO DEL TERRENO EN LA CUENCA DEL RÍO OTÚN, COLOMBIA
}

\author{
Darío Hernán Ruiz-Cobo, ${ }^{1}$ Alexander FeiJoo ${ }^{1}$ \& Carlos Rodríguez $^{2}$ \\ ${ }^{1}$ Universidad Tecnológica de Pereira, Facultad de Ciencias Ambientales. Vereda La Julita A.A. 097. \\ E-mail:dhrcobo@gmail.com, afeijoo@utp.edu.co \\ ${ }^{2}$ Universidad de La Habana, Facultad de Biología. Calle 25 \# 455 e/: J e I, Vedado, Ciudad de La \\ Habana, Cuba. E-mail: crodri@fbio.uh.cu
}

Ruiz-Cobo, D. H., A. Feijoo \& C. Rodríguez. 2010. Comunidades de marcoinvertebrados edáficos en diferentes sistemas de uso del terreno en la cuenca del Río Otún, Colombia. Acta Zoológica Mexicana (n.s.), Número Especial 2: 165-178.

RESUMEN. En los sistemas montañosos andinos algunos agrosistemas son ambientes propicios para la conservación de la biodiversidad o para la readaptación, recolonización y multiplicación natural de las especies. Para evaluar este fenómeno, en la cuenca del río Otún, Colombia se caracterizó la comunidad de macroinvertebrados edáficos en diferentes sistemas de uso del terreno. Se identificaron 19 órdenes con riqueza media estimada entre 12 y 16 grupos; el relicto de selva tuvo mayor y las plantaciones menor riqueza. Las lombrices de tierra fueron el grupo dominante en abundancia $\left(620\right.$ ind. $\left.\mathrm{m}^{-2}\right)$ y biomasa (134,1 g.p.f. $\mathrm{m}^{-2}$ ). Se encontró los valores más altos de diversidad y una distribución más homogénea y frecuente de los macroinvertebrados en el relicto de selva, así como alto porcentaje de dominancia y porcentaje de dominancia combinada (PDC) para determinados grupos epígeos como diplópodos, isópodos y arácnidos, que se consideraron indicadores de ecosistemas más conservados. El Análisis Factorial de Correspondencia para abundancia de macroinvertebrados separó significativamente $(\mathrm{p}<0.01)$ los sistemas de acuerdo al gradiente de uso y el nivel de intervención, y los dos primeros componentes explicaron el $51 \%$ de la variabilidad total.

Palabras clave: Macrofauna del suelo, uso del terreno, análisis factorial de correspondencia, dominancia combinada

Ruiz-Cobo, D. H., A. Feijoo \& C. Rodríguez. 2010. Soil macro-invertebrate communities in different land use systems in the Otún River Valley, Colombia. Acta Zoológica Mexicana (n.s.), Número Especial 2: 165-178.

ABSTRACT. In the Andean Mountains some agroecosystems are adequate environments for biodiversity conservation or for the readaptation, recolonization and natural recovery of species. To evaluate this phenomenon, soil macroinvertebrate communities were studied in different land uses of the Otún watershed in Colombia. Nineteen orders were found, with richness ranging from 12 to 16 groups per land use system; the forest had the highest while the plantations had the lowest diversity. Earthworms were the dominant group in terms of abundance (620 individuals $\mathrm{m}^{-2}$ ) and biomass (134.1 g.f.w. $\left.\mathrm{m}^{-2}\right)$.

Recibido: 16/05/2008; aceptado: 08/01/2010. 
Highest diversity, frequency and homogeneity of distribution of the soil macrofauna were observed in the forest, together with a high percentage dominance and mixed dominance for certain epigeic groups such as millipedes, isopods and arachnids, considered indicators of more preserved ecosystems. The factorial correspondence analysis for macroinvertebrate abundance significantly separated $(p<0.01)$ the systems according to land use gradient and intervention level, with the first two components explaining $51 \%$ of the total variability.

Keywords: Soil macrofauna, land use, factorial correspondence analysis, combined dominance

\section{INTRODUCCIÓN}

La cordillera de los Andes neotropicales propicia la formación de diversos hábitats aislados gracias a la altura, la irregularidad de las cadenas de montañas, las variaciones en la topografía y las pendientes, los cambios del clima y en los suelos y el rico sistema hidrográfico. En varias publicaciones se ha registrado la reducción de la biodiversidad como consecuencia de la fragmentación de las selvas andinas para la introducción de sistemas de cultivo, plantaciones forestales y pastizales (Cavelier \& Etter 1995, Van der Hammen 1995, Feijoo et al. 1999, Feijoo et al. 2005), pero poco se ha discutido acerca de la capacidad de adaptación de las formas de vida al cambio en el uso de los terrenos, para diferenciarlos individualmente o como mosaico paisajístico según su hospitalidad-hostilidad para la macrofauna edáfica.

En Colombia las superficies de protección de la vida silvestre nacieron en 1948 con la declaración como reserva nacional de la Serranía de la Macarena y crecieron hasta conformar el Sistema de Parques Nacionales Naturales constituido por 49 áreas protegidas que cubren 10.320.225 hectáreas (Inderena 1986). En el departamento de Risaralda existen dos áreas protegidas: el Santuario de Fauna y Flora Otún-Quimbaya y el Parque Regional Natural Ucumarí y aunque la adecuación de áreas de protección ha mejorado las dinámicas de las sucesiones en su interior, no se han diseñado estrategias para el vecindario que minimicen el estrés permanente ocasionado por sistemas industrializados. Estas zonas de vecindad se caracterizan por alta biodiversidad de lauráceas, vegetación de matorrales, riparia o de bosques de reforestación. En la fauna se han registrado 300 especies de aves, 58 de mamíferos, 18 de ranas y 13 de ofidios; entre los macroinvertebrados se han documentado 150 de mariposas diurnas y algunos dulce-acuícolas (PNNC 2004).

Mientras la importancia de los macroinvertebrados en la fertilidad natural de los suelos y la relación con otras comunidades edáficas se ha reconocido y valorado con biomas templados (Lavelle \& Spain 2001), son incipientes las investigaciones en el neotrópico andino. Es necesario monitorear los cambios que ocurren con la tala de la vegetación arbórea de las selvas y la sustitución por arbustos o hierbas cultivadas. También se necesita observar en el largo plazo la degradación-recuperación de la calidad del suelo en relación con los itinerarios técnicos de los sistemas de cultivo y de crianza (Feijoo et al. 1999, Baretta et al. 2006). 
Por todo lo anterior, se propuso realizar el primer acercamiento a la caracterización de los macroinvertebrados edáficos en diferentes usos del terreno en la cuenca del río Otún y diferenciar los hábitat de acuerdo con el nivel de intensificación del manejo antrópico. Se planteó como hipótesis que los cambios propiciados en el suelo como consecuencia de la acentuación de la intensidad de uso de los terrenos tropandinos se pueden leer con los cambios en la estructura de la macrofauna del suelo.

\section{Descripción del área de estudio \\ MATERIAL Y MÉTODOS}

El estudio se realizó en mosaicos del paisaje sobre usos del terreno con intervención humana y en áreas conservadas durante más de 50 años en la vertiente occidental de la cordillera Central, en el corregimiento de la Florida, el Parque Regional Natural Ucumarí y el Santuario de Flora y Fauna Otún-Quimbaya, parte media de la cuenca del río Otún, departamento de Risaralda, Colombia (Fig. 1). La investigación se

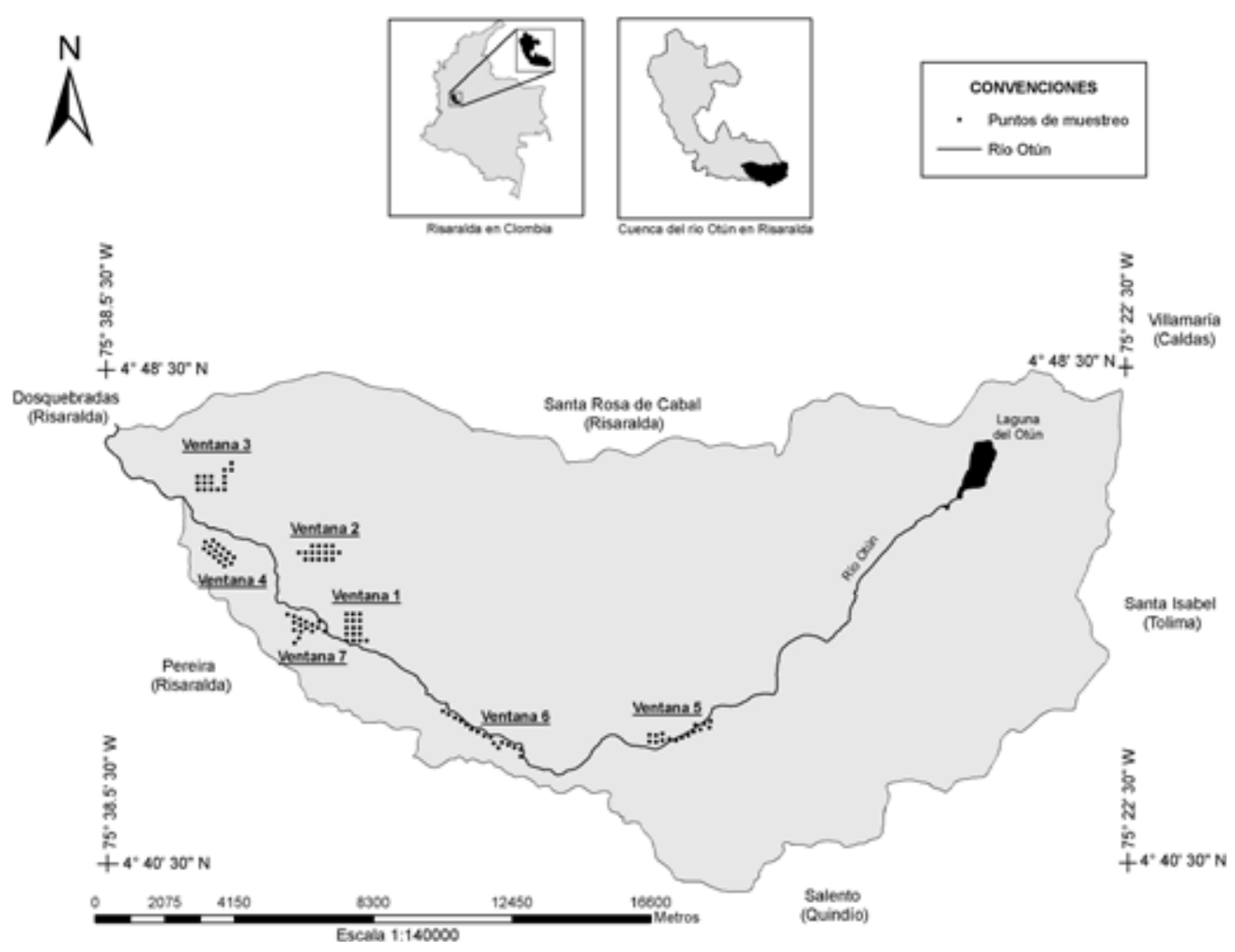

Figura 1. Localización de las 7 ventanas de $1 \mathrm{~km}^{2}$ en la parte media de la cuenca del río Otún, Colombia. 
adelantó entre junio y septiembre de 2006 en el segundo periodo seco e inicio del segundo periodo lluvioso, en los subsistemas hídricos de captación y de transporte (Hamblin \& Christiansen, 1998) entre 1650 y $2500 \mathrm{msnm}$. En la región de estudio la temperatura media es de $14-18^{\circ} \mathrm{C}$ y la precipitación de $2700 \mathrm{~mm} / \mathrm{año}$, perteneciendo a las zonas de vida Sub-andina y Andina (Cuatrecasas 1958, Rangel \& Aguilar 1994).

Los suelos derivados de ceniza volcánica varían desde ferralíticos moderados o jóvenes (oxic Dystropept; Dystrandept, Hapludalf; Tropudult) en la zona sub-andina con arcillas 2:1 y 1:1 hasta los Andosoles (Humitropept, Hapludent, Dystropept) en la andina, ricos en materia orgánica con formación masiva de alofana (silicatos de aluminio mal cristalizados) y de productos amorfos (gels de $\mathrm{Si}, \mathrm{Al}$ y FeO) (Thouret 1989).

\section{Selección de sitios para el estudio}

Se situaron siete ventanas de $1 \mathrm{~km}^{2}$ y se geo-referenciaron 16 puntos separados a $200 \mathrm{~m}$ (Fig. 1). El diseño utilizado fue sistemático, estratificado en dos dimensiones (modelo de red cuadrada), con la selección de un par de números al azar para fijar las coordenadas de la unidad superior izquierda y disminuir los efectos de la auto correlación (Cochran 1974); además este método permitió construir la ventana alineada o desalineada de manera bidimensional, de acuerdo con los cambios en la topografía o en el paisaje e información obtenida en proyectos previos de otros programas internacionales (Mathieu et al. 2005). Los muestreos se llevaron a cabo en pastizales, monocultivos de café (Coffea arabica), cultivos de café asociado con plátano (Musa sp.) y guadua (Guadua angustifolia), cultivos de yuca (Manihot esculenta) y maíz (Zea mays), plantas medicinales, plantaciones de ciprés (Cupressus sempervirens), eucalipto (Eucalyptus sp.), guadua, pino (Pinus patula) y urapán (Fraxinus chinensis), en sucesiones arbustivas y arbóreas y en relictos de selva.

\section{Muestreo de las variables del suelo}

Para los macroinvertebrados (animales visibles a la vista, de diámetro $>2 \mathrm{~mm}$ o longitud $>10 \mathrm{~mm}$ ) se siguió la metodología del programa internacional Tropical Soil Biology and Fertility (TSBF) (Anderson \& Ingram 1993). En cada punto se separó un volumen de suelo de $25 \times 25 \times 30 \mathrm{~cm}$ de profundidad con una zanja alrededor de $30 \mathrm{~cm}$ para aislar y extraer de forma manual los macroinvertebrados (excepto insectos sociales) en 0-10, 10-20 y 20-30 cm y conservarlos en alcohol al 70\% y las lombrices de tierra en formol al 5\%. Posteriormente los organismos se identificaron a nivel de orden, familia, o especie y se cuantificó la abundancia (ind. $\mathrm{m}^{-2}$ ) y la biomasa (g.p.f. $\mathrm{m}^{-2}$ ).

\section{Análisis de la información}

Los registros de los usos y coberturas de los terrenos se agruparon para reducir la variabilidad de la información y se ordenaron en cinco categorías: relicto de selva (Rls, 
$\mathrm{n}=17$ ), plantaciones forestales ( $\mathrm{Pln}, \mathrm{n}=24)$, sucesiones (Suc, $\mathrm{n}=27)$, cultivos (Cul, $\mathrm{n}=8$ ) y pastizales (Pas, $\mathrm{n}=36$ ).

Los datos se analizaron con las pruebas de Kolmogorov-Smirnov (normalidad) y Levene (homogeneidad de varianza) con el programa InfoStat/Profesional V.2006d. La estimación de la riqueza esperada de la fauna se analizó con el programa Estimate S V. 7.5.1, que permitió calcular las curvas de saturación para conocer la confiabilidad del esquema de muestreo. Las diferencias entre usos se calcularon con ANOVA simple y la prueba de Tukey o análisis de varianza no paramétrico (Kruskal-Wallis).

Para determinar los grupos zoológicos de mayor importancia en los sistemas de uso de los terrenos, se calcularon los porcentajes de frecuencia de aparición (PF), abundancia (PA) y biomasa (PP); así como el porcentaje de dominancia combinada (PDC) ya que en ocasiones los primeros no reúnen los valores máximos en un mismo grupo (Jesús et al. 1981).

En la escala del paisaje se calculó la frecuencia de aparición, abundancia, biomasa para los 112 monolitos y los 13 usos del terreno y se redujo la variabilidad de la información en función de las variables de los macroinvertebrados con el Análisis Factorial de Correspondencia (AFC) (ADE-4 V.2001) (Thioulouse et al. 1997).

\section{RESULTADOS}

\section{Composición taxonómica y riqueza}

Se colectaron 4339 individuos de macroinvertebrados distribuidos en tres Phyla, ocho clases y 19 órdenes. Se separaron 37 morfotipos y 12 familias de diplópodos, 15 familias de coleópteros y 15 especies de lombrices de tierra.

La riqueza media según el método de Jackknife (Fig. 2) mostró diferencias significativas entre los usos del terreno $(\mathrm{p}<0,05)$; los relictos de selva presentaron la mayor riqueza $(15,9)$ y mostraron diferencias altamente significativas con los demás sistemas. Los pastizales, sucesiones y cultivos no se diferenciaron $(15,1-15,3)$. El valor más bajo de riqueza se encontró en las plantaciones $(13,6)$ con diferencias altamente significativas con relación a los restantes, lo cual se atribuyó probablemente a los bajos valores encontrados en las plantaciones de pino y de ciprés (entre 4 y 14).

\section{Dominancia}

Los macroinvertebrados de mayor presencia fueron las lombrices de tierra, los estados larvales de insectos, diplópodos (con presencia en el 50\% de las muestras) y en menor medida los coleópteros (Cuadro I). Los dos primeros grupos se catalogaron como "absolutamente constantes" ( $>75 \%$ de frecuencia), los diplópodos como "constantes" (50-75\%) y coleópteros, quilópodos, isópodos y arácnidos como "accesorios" (25-50\%). Los restantes fueron clasificados como "accidentales" $(<25 \%)$. Lombrices de tierra, larvas de insectos y diplópodos presentaron diferencias altamente significa- 


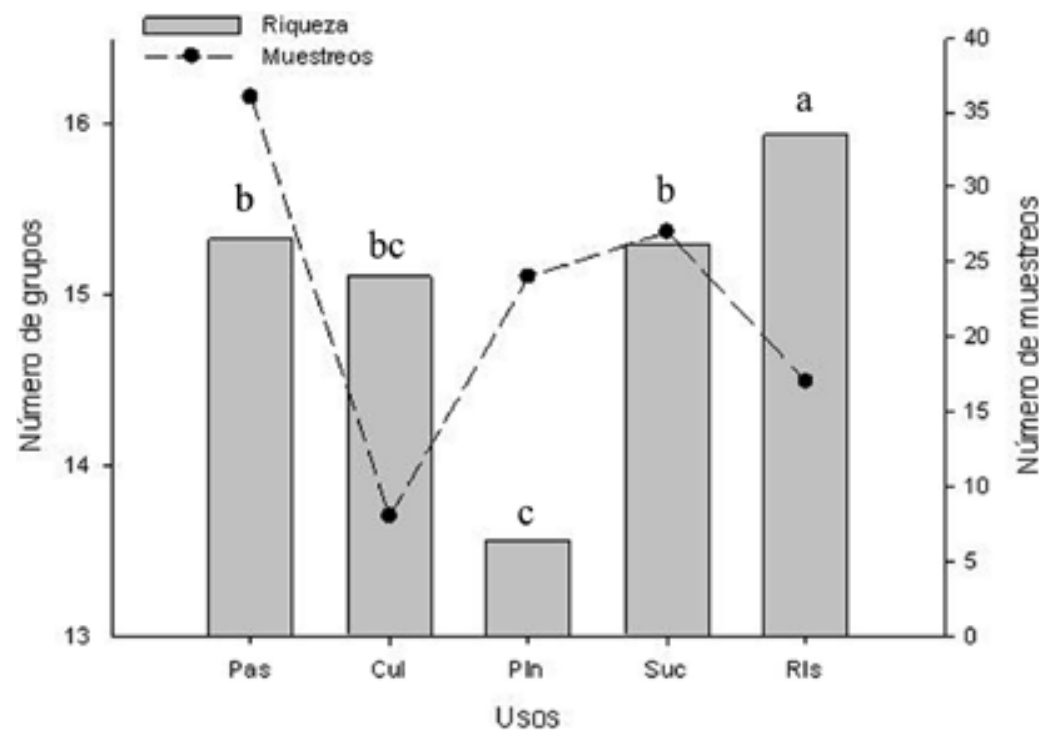

Figura 2. Riqueza promedio estimada según el método de Jackknife y número de muestreos en los diferentes sistemas de uso de la tierra en la parte media de la cuenca del río Otún, Colombia. Letras distintas indican diferencias significativas al nivel $\mathrm{p}<0,05$.

tivas en abundancia y biomasa $(\mathrm{p}<0,01)$. El valor más elevado del PDC correspondió a las lombrices de tierra $(>50 \%)$, larvas de insectos y diplópodos pero con valores inferiores.

El relicto de selva y las sucesiones presentaron el mayor número de grupos zoológicos con valores de frecuencia de aparición relativamente altos ( $>50 \%)$ por lo tanto, son los sistemas que exhiben la distribución horizontal más amplia y heterogénea con alta presencia de grupos epígeos como arácnidos e isópodos (42 y 61\%); los coleópteros se presentaron con mayor frecuencia en cultivos (75\%), mientras las lombrices de tierra y larvas de insectos dominaron en todos los sistemas. Para los porcentajes de abundancia, biomasa y el PDC, las lombrices de tierra dominaron en todos los sistemas de uso del terreno aunque con valores más bajos en los bosques donde el aporte de diplópodos y larvas de insectos aumentó (Cuadro I).

\section{Abundancia y biomasa}

La comunidad de macroinvertebrados presentó una abundancia media de 620 ind. $\mathrm{m}^{-2}$ con una biomasa de 134.1 g.p.f. $\mathrm{m}^{-2}$. Los grupos más abundantes fueron los isópodos, diplópodos y larvas de insectos en los relictos de selva y las sucesiones, y las lombrices de tierra en pastizales y cultivos (Cuadro II). 
Cuadro I. Índice de dominancia para los macroinvertebrados colectados en diferentes sistemas de uso del terreno y el promedio de todas las muestras (nivel de paisaje), en la parte media de la cuenca del río Otún, Colombia.

\begin{tabular}{|c|c|c|c|c|c|c|c|c|c|c|c|c|}
\hline Usos & Olig & Col & Dip & Chi & Der & Bla & Iso & Ort & Hem & Ara & Lar & Otr \\
\hline \multicolumn{13}{|c|}{ Pastizal } \\
\hline $\mathrm{PF}$ & 97 & 25 & 19 & 3 & 6 & 6 & 14 & 0 & 11 & 11 & 69 & 6 \\
\hline $\mathrm{PA}$ & 89 & 1 & 1 & $<1$ & $<1$ & $<1$ & 1 & 0 & $<1$ & $<1$ & 6 & $<1$ \\
\hline PB & 94 & $<1$ & $<1$ & $<1$ & $<1$ & $<1$ & $<1$ & 0 & $<1$ & $<1$ & 5 & $<1$ \\
\hline PDC & 73 & 4 & 3 & $<1$ & 1 & 1 & 2 & 0 & 2 & 2 & 7 & $<1$ \\
\hline \multicolumn{13}{|c|}{ Cultivos } \\
\hline $\mathrm{PF}$ & 100 & 75 & 63 & 38 & 0 & 13 & 25 & 0 & 38 & 13 & 88 & 25 \\
\hline PA & 71 & 4 & 4 & 1 & 0 & 1 & 1 & 0 & 3 & 1 & 13 & 1 \\
\hline PB & 91 & 1 & 4 & $<1$ & 0 & $<1$ & $<1$ & 0 & $<1$ & $<1$ & 4 & $<1$ \\
\hline PDC & 61 & 7 & 7 & 3 & 0 & 1 & 2 & 0 & 4 & 1 & 12 & 2 \\
\hline \multicolumn{13}{|c|}{ Plantaciones } \\
\hline $\mathrm{PF}$ & 96 & 38 & 29 & 33 & 4 & 21 & 11 & 4 & 29 & 25 & 92 & 8 \\
\hline PA & 64 & 3 & 6 & 2 & $<1$ & 2 & 2 & $<1$ & 2 & 2 & 17 & 2 \\
\hline PB & 93 & 1 & 1 & $<1$ & $<1$ & $<1$ & $<1$ & $<1$ & 1 & $<1$ & 2 & $<1$ \\
\hline PDC & 60 & 4 & 5 & 3 & $<1$ & 3 & 2 & $<1$ & 3 & 3 & 14 & 1 \\
\hline \multicolumn{13}{|c|}{ Sucesión } \\
\hline $\mathrm{PF}$ & 100 & 58 & 77 & 42 & 19 & 27 & 62 & 12 & 15 & 42 & 100 & 23 \\
\hline $\mathrm{PA}$ & 47 & 3 & 13 & 4 & 1 & 1 & 8 & $<1$ & 1 & 1 & 20 & 2 \\
\hline PB & 87 & $<1$ & 3 & $<1$ & $<1$ & $<1$ & 1 & $<1$ & $<1$ & $<1$ & 8 & $<1$ \\
\hline PDC & 50 & 4 & 10 & 4 & 1 & 2 & 6 & 1 & 1 & 3 & 15 & 1 \\
\hline \multicolumn{13}{|c|}{ Relicto de Selva } \\
\hline $\mathrm{PF}$ & 94 & 41 & 100 & 65 & 35 & 41 & 59 & 29 & 12 & 53 & 88 & 12 \\
\hline PA & 30 & 2 & 20 & 4 & 1 & 1 & 7 & 1 & $<1$ & 4 & 29 & 1 \\
\hline PB & 54 & 1 & 8 & 1 & 1 & $<1$ & 2 & $<1$ & $<1$ & $<1$ & 32 & $<1$ \\
\hline PDC & 33 & 3 & 14 & 5 & 3 & 3 & 6 & 2 & 1 & 4 & 21 & 1 \\
\hline \multicolumn{13}{|c|}{ Promedio } \\
\hline $\mathrm{PF}$ & 96 & 41 & 50 & 30 & 13 & 20 & 34 & 8 & 18 & 28 & 85 & 11 \\
\hline PA & 66 & 2 & 7 & 2 & 1 & 1 & 4 & $<1$ & 1 & 1 & 15 & 1 \\
\hline PB & 70 & $<1$ & 1 & $<1$ & $<1$ & $<1$ & $<1$ & $<1$ & $<1$ & $<1$ & 6 & $<1$ \\
\hline PDC & 53 & 4 & 7 & 3 & 1 & 2 & 4 & 1 & 2 & 3 & 14 & 1 \\
\hline
\end{tabular}

Olig: Oligochaeta, Col: Coleoptera, Dip: Diplopoda, Chi: Chilopoda, Der: Dermaptera, Bla: Blattodea, Iso: Isopoda, Ort: Orthoptera, Hem: Hemiptera, Ara: Arachnida, Lar: Larvas, Otr: Otros. (PF): Porcentaje de frecuencia de aparición, (PA): Porcentaje de abundancia, (PB): Porcentaje de biomasa, (PDC): Porcentaje de dominancia combinada.

El análisis de abundancia y biomasa totales por sistema no mostró diferencias significativas entre los usos del terreno; sin embargo la separación por grupos de macroinvertebrados mostró algunas diferencias significativas, especialmente para los 


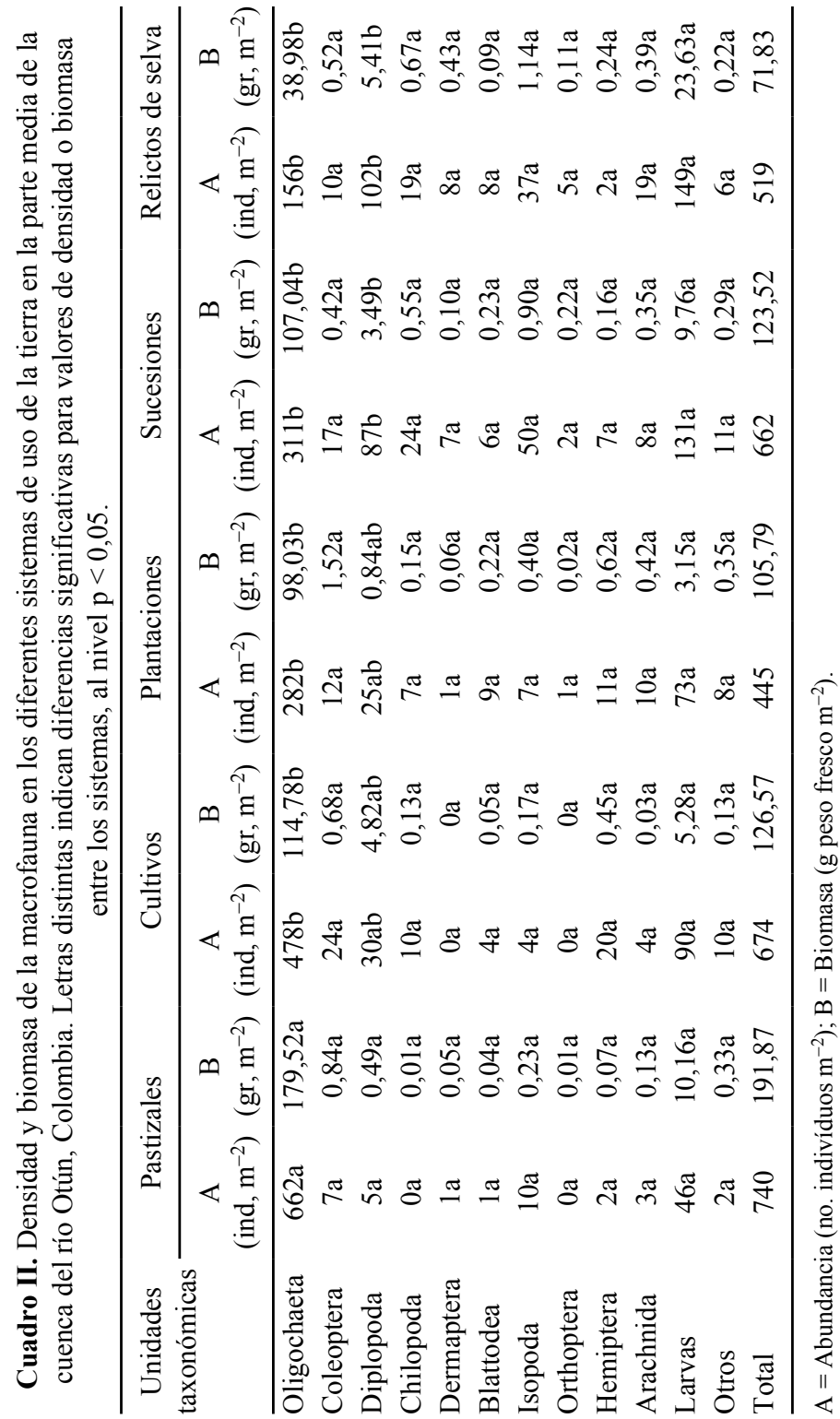


pastizales en comparación a los demás sistemas. Se encontraron diferencias significativas $(p<0,05)$ en la abundancia y biomasa de lombrices en los pastizales con respecto a los demás sistemas de uso del terreno; en densidad y biomasa de diplópodos en pastizales con relación a los sistemas menos intervenidos por el hombre (relicto de selva y sucesión) (Cuadro II).

El AFC para abundancia de macroinvertebrados separó significativamente $(\mathrm{p}<0,01)$ los sistemas de acuerdo con el gradiente de uso y el nivel de intervención (Fig. 3); los dos primeros componentes explicaron el 51\% de la variabilidad total ( $33 \%$ y $18 \%$ para las componentes 1 y 2 , respectivamente). Los sistemas se alinearon a lo largo del primer eje, con los sistemas más conservados concentrándose del lado izquierdo y los mas perturbados del lado derecho. Los grupos epígeos predadores y saprófagos (Isopoda, Arachnida, Diplopoda, Chilopoda, Blattodea y Dermaptera) estuvieron asociados a hábitats más conservados con vegetación diversificada (sucesiones y los relictos de selva) y quedaron en oposición a las lombrices de tierra exóticas y nativas (Pontoscolex corethrurus, Amynthas corticis y Glossodrilus sp.) y los hemípteros, asociados a los ambientes más perturbados (pastizales, cultivos y plantaciones).
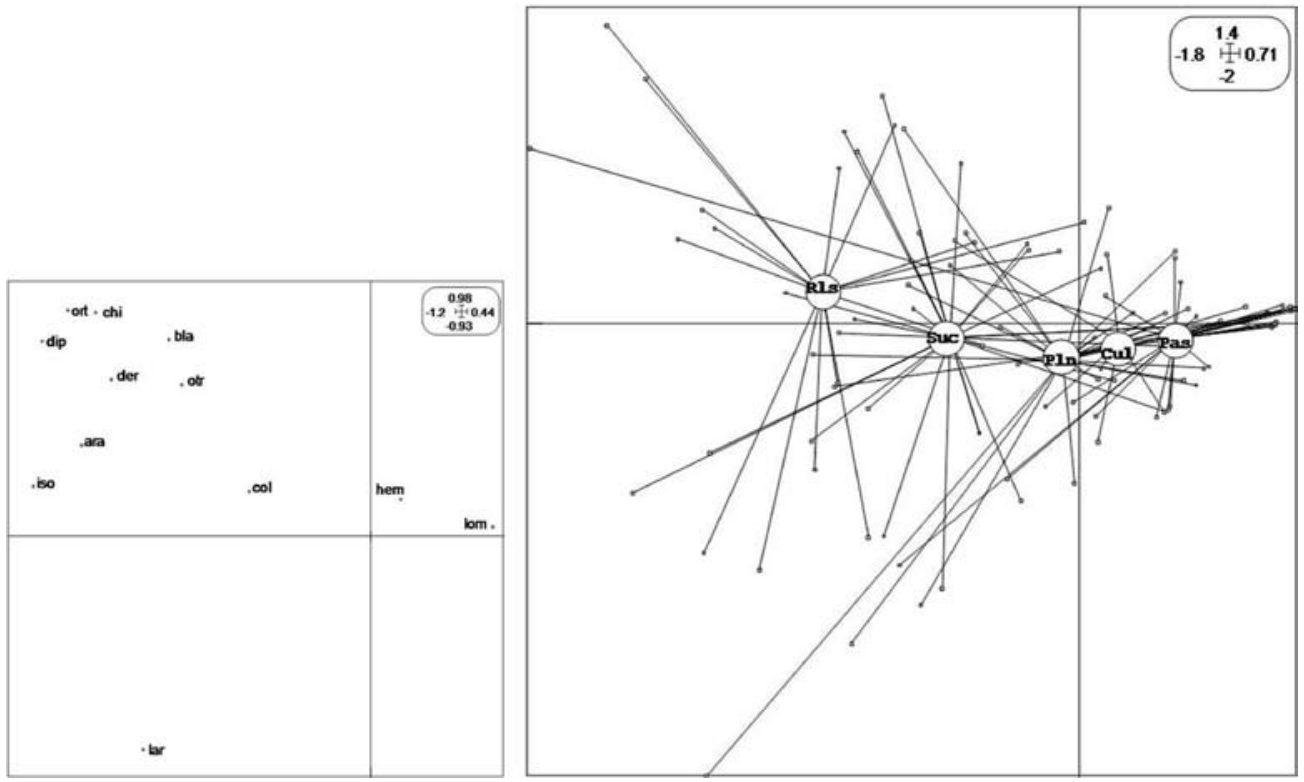

Figura 3. Proyección de las variables y los sitios de muestreo en el plano factorial con dos factores extraídos en el AFC de la densidad de la macrofauna en la parte media de la cuenca del río Otún, Colombia $(\mathrm{P}<0.001)$. ort $=$ Orthoptera, dip $=$ Diplopoda, chi $=$ Chilopoda, der $=$ Dermaptera, bla $=$ Blattodea, otr $=$ Otros, ara $=$ Arachnida, iso $=$ Isopoda, $\mathrm{col}=$ Coleoptera, hem $=$ Hemiptera, lar $=$ Larvas, oli $=$ Oligochaeta. 
En la biomasa el AFC explicó el $62,8 \%$ de la variabilidad total. El primer componente $(45,7 \%)$ agrupó sistemas con baja biomasa proveniente de diversos grupos de macroinvertebrados asociados con relictos de selva, mientras que el segundo (17\%) agrupó usos del terreno con más intervención (plantaciones, cultivos y pastizales) y biomasa más alta, con predominio de lombrices de tierra y coleópteros.

\section{DISCUSIÓN}

En los sistemas trop-andinos algunos sistemas tradicionales como cafetales con sombrío o maíz y fríjol ofrecen ambientes propicios para la conservación de la biodiversidad, recolonización y multiplicación de las especies y han emergido como islas biogeográficas o refugios críticos en medio de paisajes transformados.

La riqueza de las comunidades de macroinvertebrados en la cuenca media del río Otún varió de acuerdo con el gradiente de intensificación de uso y nivel de perturbación del sistema y la cobertura vegetal (y consecuente aporte de hojarasca de la vegetación). Los valores de riqueza fluctuaron con tendencias similares a los encontrados en otros estudios en los Andes colombianos, realizados en selvas, barbechos, cultivos y pastizales en Caldono, Cauca (Feijoo et al. 1999), en sistemas sin y con intervención en la cuenca del río La Vieja (Feijoo et al. 2005) y en sistemas de cultivo, pastos y bosque en la microcuenca de Potrerillo, Cauca (Velásquez et al. 2007). También fueron similares a los resultados de investigaciones en ambientes de selva amazónica (Barros et al. 2003) y en diferentes sistemas de uso en el paleo-trópico hindú (Rossi \& Blanchart 2005).

La perturbación del ecosistema suelo por labranza (Marín \& Feijoo 2007), el sistema de cultivo en surcos continuos, la aplicación de insumos de síntesis química (Brown et al. 2004, Sileshi \& Mafongoya 2006, Velásquez 2007), y la introducción de plantaciones con especies exóticas influye de manera directa sobre la abundancia de las poblaciones de macroinvertebrados. La baja riqueza de especies presente en las plantaciones de pino y ciprés puede estar asociada con la homogeneidad de la hojarasca, la composición química (González et al. 1999), la presencia de sustancias tóxicas (Perera 1992) y a la resistencia mecánica a la masticación (Bengtsson et al. 1997).

En frecuencia, biomasa y PDC las lombrices de tierra fueron dominantes, por lo que la contribución al establecimiento estructural y funcional de la comunidad y del paisaje debe ser decisiva. Diferentes autores coinciden en señalar que la dominancia de las lombrices de tierra en la biomasa en los ecosistemas tropicales tiene efecto prominente en las propiedades del suelo al influir en procesos físicos y biogeoquímicos esenciales para el crecimiento de las plantas (Anderson \& Flanagan 1989, Blair et al. 1995, Brussaard et al. 1997).

La más homogénea y amplia distribución horizontal de un mayor número de grupos de macroinvertebrados catalogados como "constantes" y "absolutamente cons- 
tantes" presente en los relictos de selva y sucesiones, así como la disminución que sufren los porcentajes de dominancia numérica, en biomasa y PDC de lombrices y la elevación de estos índices para otros grupos como los artrópodos (diplópodos y larvas de insectos, entre otros), manifiestan en alguna medida mayor equitatividad en dichos ecosistemas. Esta condición es de gran importancia debido a la posible función reguladora de la biodiversidad sobre la estabilidad de la comunidad y su contribución a la dinámica ecológica y resistencia al estrés en los ecosistemas (de Oliveira et al. 2005). La heterogeneidad del hábitat y la variabilidad florística amplían el espectro alimentario y de microhábitats para la comunidad, favorecido además por el depósito de abundante hojarasca que actúa como aislante y conserva la temperatura y la humedad. Por lo tanto, no es de sorprenderse que en estos sistemas grupos epígeos como arácnidos e isópodos, usualmente asociados con hábitat más diversos (de Oliveira et al. 2005) presentaron alta frecuencia.

Los procesos de conversión de la selva andina a sistemas agrícolas pasando por la formación de pastizales se asocian con el predominio de pocas lombrices exóticas, lo cual conlleva al incremento de la densidad y biomasa de la comunidad de lombrices. Las asociaciones encontradas entre las lombrices de tierra con los pastizales, cultivos y plantaciones, así como las de isópodos, arácnidos y coleópteros con los sistemas de menor efecto antrópico (relictos y sucesiones) ameritan el desarrollo de estudios complementarios con mayor detalle taxonómico para verificar la diversidad específica en estos ecosistemas, la presencia de posibles especies indicadores de calidad de los agroecosistemas y la contribución de estos macroinvertebrados a los servicios ambientales y a la multifuncionalidad de la agricultura.

El análisis de AFC fue significativo y separó usos del suelo de acuerdo con el gradiente de intensidad; la herramienta permitió separar los efectos relacionados con la fragmentación de la selva andina con las áreas de mayor conservación (alta riqueza de grupos) o presencia de mezclas de especies de lombrices exóticas y nativas en ambientes cultivados, plantaciones y pastizales. Las comunidades de macroinvertebrados fueron afectadas de manera diferencial, positiva o negativa, por la conservación del sombrío y los aportes de coberturas, mantenimiento de relictos de selva para la conservación del agua y la biodiversidad y por algunas prácticas de manejo de los agroecosistemas que no produjeron daños profundos al introducir parches con agricultura campesina de subsistencia o sistemas agroindustriales.

La introducción de dichas prácticas de manejo (modificación de ambientes naturales, movimientos de tierra, arreglos variados en los agroecosistemas, cultivos en surco con aplicación de agroquímicos, intensificación de pastizales) impactan la conectividad incidiendo en la modificación de los mapas de los biotopos, la hemerobia local (Zebish et al. 2004), lo cual repercute en la composición, estructura y funciones múltiples. Estos procesos de transformación incluyeron la detección de agrosistemas con diverso manejo y características de sitio asociadas con la intervención humana, 
que produce cambios en hábitats permitiendo la readaptación de la vida a otros sistemas con múltiples tipos de parches, calidad de hábitat y abundancia variable en partes diferentes del mosaico (Bennett et al. 2006), vecindario (Hersperger 2006) o proveer resiliencia ecológica, lo cual es pobremente documentado (Jackson et al. 2007).

Para dar continuidad a las siguientes fases de éste proyecto se requiere correlacionar los procesos y la dinámica a nivel del paisaje con actividades agrícolas, formas de manejo, áreas construidas, cualidades del suelo, registros de biomasa vegetal, carbono, nitrógeno, las áreas de los usos del suelo y la abundancia de lombrices para empezar a diseñar una matriz que posibilite evaluar las interacciones e introducir los conceptos de sistemas humanos para calificar y organizar pronósticos que planifiquen el uso del terreno. Así mismo se necesita localizar las variaciones en las consecuencias de las interacciones entre subsistemas humanos y naturales con análisis específicos y el empleo de ciencias de la información geográfica, con la participación de grupos multidisciplinarios de las ciencias naturales, sociales y espaciales (Rindfuss et al. 2004).

\section{AGRADECIMIENTOS}

Este trabajo fue parte de la tesis de maestría del primer autor en la Universidad de La Habana, Cuba. Los autores agradecen al Centro de Investigaciones y Estudios en Biodiversidad y Recursos Genéticos (CIEBREG) y a Colciencias por la financiación del proyecto "Valoración de los bienes y servicios de la biodiversidad para el desarrollo sostenible de paisajes rurales colombianos, Complejo Ecorregional de los Andes del Norte (CEAN)", a la Universidad Tecnológica de Pereira por el apoyo para la realización de la investigación. Al maestro Heimar Quintero Vargas por el apoyo y recomendaciones y Elena Velásquez por la ayuda en el análisis factorial de correspondencia, a Jorge Marulanda y la empresa Aguas y Aguas de Pereira por facilitar los predios para llevar a cabo los muestreos.

\section{LITERATURA CITADA}

Anderson, J. M. \& P. W. Flanagan. 1989. Biological processes regulating organic matter dynamics in tropical soils. Pp. 97-122. In: D.C. Coleman, J.M. Oades and G. Uehara (Eds). Dynamics of soil organic matter in tropical ecosystems. University of Hawaii Press, Honolulu.

Anderson, J. M. \& J. S. I. Ingram. 1993. Tropical soil biology and fertility: A handbook of methods, $2^{\text {nd }}$ Ed. CAB International, Wallingford.

Baretta, D., A. L. Mafra, J. C. Pires Santos, C. Vidal \& I. Bertol. 2006. Análise multivariada da fauna edáfica em diferentes sistemas de preparo e cultivo do solo. Pesquisa Agropecuária Brasileira. 41(11): 1675-1679.

Barros, E., A. Neves, E. Blanchart, C. M. Fernandes, E. Wandelli \& P. Lavelle. 2003. Development of the soil macrofauna community under silvopastoral and agrosilvitultural system in Amazonia. Pedobiologia. 47: 273-280.

Bennet, A. F., J. Q. Radford \& A. Haslem. 2006. Properties of land mosaics: implications for nature conservation in agricultural environments. Biological Conservation. 133: 250-264. 
Bengtsson, J., T. Persson \& H. Lundkvist. 1997. Long-term effects of logging residue addition and removal on macroarthopods and enchytraeids. Journal of Applied Ecology. 34(4): 1014-1022.

Bernal, E., D. García, M. Novoa \& A. Pinzón. 2006. Caracterización de la comunidad de macroinvertebrados de la quebrada Paloblanco de la cuenca del río Otún (Risaralda, Colombia). Acta Biológica Colombiana. 11(2): 45-59.

Blair, J., R. W. Palmelee \& P. Lavelle. 1995. Influences of earthworms on biogeochemistry. Pp. 127158. In: P.F. Hendrix (Ed.). Earthworm ecology and biogeography in North America. Lewis, Boca Raton.

Brown, G. G., A. G. Moreno, I. Barois, C. Fragoso, P. Rojas, B. Hernández \& J. C. Patrón. 2004. Soil macrofauna in SE Mexican pastures and the effect of conversion from native to introduced pastures. Agriculture, Ecosystems and Environment. 103: 313-327.

Brussaard, L., V. S. R. Vadakattu, V. B. Behan-Pelletier, V. K. Brown, P. Lavelle, W. Didden, D. W. Malloch \& P. Folgarait. 1997. Biodiversity and ecosystem functioning in soil. Ambio. 26(8): 563-570.

Cavelier, J. \& A. Etter. 1995. Deforestation of montane forest in Colombia as a result of illegal plantations of opium (Papaver somniferum). Pp.125-137. In: S. P. Churchill, H. Balslev, E. Forero and J. L. Luteyn (Eds.). Biodiversity and conservation of Neotropical montane forests. The New York Botanical Garden, New York.

Cochran, W. G. 1974. Técnicas de muestreo, $4^{\mathrm{a}}$ Ed. Continental S. A., México.

Cuatrecasas, J. 1958. Aspectos de la vegetación natural de Colombia. Revista Académica Colombiana de Ciencias Exactas Físico - Químicas y Naturales. 10(40): 221-268.

de Oliveira, A., J. G. Marinho, R. D. Junqueira \& A. M. De Aquino. 2005. Soil macrofauna in cover crops of figs grown under organic management. Scientia Agrícola. 62(1): 5-61.

Feijoo, A., E. B. Knapp, P. Lavelle \& A. G. Moreno. 1999. Quantifying soil macrofauna in a Colombian watershed. Pedobiologia. 43: 513-517.

Feijoo, A., H. Quintero, C. Fragoso \& A. G. Moreno. 2004. Patrón de distribución y listado de especies de las lombrices de tierra (Annelida, Oligochaeta) en Colombia. Acta Zoológica Mexicana. 20(2): 197-220.

Feijoo, A., M. C. Zuñiga \& J. C. Camargo. 2005. Signs to detect regeneration and degradation of agroecosystems in the coffee growing region of Colombia. Livestock Research for Rural Development 17 (3). http://www.cipav.org.co/lrrd/lrrd17/3/feij17025.htm

González, G., X. Zou, A. Sabat \& N. Fetcher. 1999. Earthworm abundance and distribution pattern in contrasting plant communities within a tropical wet forest in Puerto Rico. Caribbean Journal of Science. 35(1-2): 93-100.

Hersperger, A. M. 2006. Spatial adjacencies and interactions: Neighborhood mosaics for landscape ecological planning. Landscape and Urban Planning. 77: 227-239.

Hamblin, W. K. \& E. H. Christiansen. 1998. Earth's dynamic systems, $8^{\text {th }}$ Ed. Prentice Hall, Upper Saddle River.

Jackson, L. E., U. Pascual \& T. Hodgkin. 2007. Utilizing and conserving agrobiodiversity in agricultural landscapes. Agriculture, Ecosystems \& Environment. 121: 196-210.

Jesús, J. B., A. G. Moreno \& D. J. Diaz Cosin. 1981. Lombrices de tierra de la Vega de Aranjuez (España). Asociaciones. Revue d'Écologie et de Biologie du Sol. 18(4): 507-519.

Lavelle, P. \& A. Spain. 2001. Soil ecology. Kluwer Academic, Dordrecht.

Marín, P., A. Feijoo. 2007. Efecto de la labranza sobre macroinvertebrados del suelo en Vertisoles de un área de Colombia. Terra Latinoamericana. 25(3): 297-310.

Mathieu, J., J. P. Rossi, M. Grimaldi, P. Mora, P. Lavelle \& C. Rouland. 2005. A multi-scale study of soil macrofauna biodiversity in Amazonian pastures. Biology and Fertility of Soils. 40: 300-305. 
Parques Nacionales Naturales de Colombia. 2004. Plan básico de manejo 2005-2009 santuario de fauna y flora Otún Quimbaya. PNNC, Medellín.

Perera, A. H. 1992. Ecological implications of establishing pine plantations in the Central region of Sri Lanka. Pp. 409-414. In: K. P. Singh and J. S. Singh (Eds.). Tropical ecosystems: ecology and management. Wiley Eastern, New Delhi.

Rangel, J. O. \& M. Aguilar. 1994. Una aproximación sobre la diversidad climática en las regiones naturales de Colombia. Pp. 25-76. In: J. O. Rangel (Ed.). Colombia diversidad biótica I. Universidad Nacional de Colombia, Bogotá.

Rindfuss, R. R., S. J. Walsh, B. L. Turner, J. Fox \& V. Misra. 2004. Development a science of land change: Challenges and methodological issues. Proceedings of the National Academy of Sciences of the United States of America. 101(39): 13976-13981.

Rossi, J. P. \& E. Blanchart. 2005. Seasonal and land-use induced variations of soil macrofauna composition in the Western Ghats, southern India. Soil Biology \& Biochemistry. 37: 1093-1104.

Sileshi, G. \& P. P. Mafongoya. 2006. Variation in macrofaunal communities under contrasting land use systems in Eastern Zambia. Applied Soil Ecology. 33: 49-60.

Thouret, J. C. 1989. Suelos de la Cordillera Central, transecto Parque de Los Nevados. Pp. 293-441. In: T. van der Hammen, S. Díaz-Piedrahita and V. J. Alvarez (Eds.). Studies on tropical Andean ecosystems, Vol.3. J. Cramer, Berlin.

Thioulouse, J, D. Chessel, S. Dolédec \& J. M. Olivier. 1997. ADE-4: A multivariate analysis and graphical display software. Statistical Computation. 7: 2-15.

Van der Hammen, T, S. Díaz-Piedrahita \& V. J. Alvarez. 1989. Studies on tropical Andean ecosystems, Vol. 3. J. Cramer, Berlin.

Van der Hammen, T. 1995. Global change, biodiversity and conservation of Neotropical montane forests. Pp. 603-607. In: S. Churchill, H. Balslev, E. Forero and J. Luteyn (Eds.). Biodiversity and conservation of Neotropical montane forests. The New York Botanical Garden, New York.

Velasquez, E., P. Lavelle \& M. Andrade. 2007. GISQ a multifunctional indicator of soil quality. Soil Biology \& Biochemistry. 39: 3066-3080.

Zebisch, M., F. Wechsung \& H. Kenneweb. 2004. Landscapes response functions for biodiversity - assessing the impact of land-use changes at the county level. Landscape and Urban Planning. 67: $157-172$. 